ECLETICA A A

$\underline{\text { www.scielo.br/eq }}$

\title{
Determinação voltamétrica do nedocromil de sódio utilizando eletrodo de carbono vítreo
}

\author{
T. F. Rosal, A. A. Saczk ${ }^{1}$, M. V. B. Zanoni ${ }^{1}$, N. R. Stradiotto ${ }^{1}$ \\ ${ }^{1}$ Departamento de Química Analítica - Instituto de Química - UNESP - 14801-970 - Araraquara - SP - Brasil
}

Resumo: Estudos eletroanalíticos foram realizados com o Nedocromil de Sódio empregando as técnicas voltamétricas de varredura linear, pulso diferencial e onda quadrada, em tampão Britton-Robinson ( $\mathrm{pH}$ $4,0)$. No estudo voltamétrico empregando a modalidade de varredura linear observou-se dois picos catódicos, irreversíveis, nos valores de potencial de - $0,86 \mathrm{~V}$ e $-1,10 \mathrm{~V}$ (vs ECS). As correntes de pico catódicas apresentaram um controle difusional segundo a relação $I_{p c}$ versus $v^{1 / 2}$. A dependência de $I_{p c}$ com a concentração apresentou linearidade entre $5,0 \times 10^{-4} \mathrm{~mol} \mathrm{~L}^{-1}$ e $5,0 \times 10^{-3} \mathrm{~mol} \mathrm{~L}^{-1}$, limite de deteç̧ão de $8,2 \times 10^{-5} \mathrm{~mol} \mathrm{~L}^{-1}$ e sensibilidade de $6,3 \times 10^{3} \mathrm{~mA} / \mathrm{mol} \mathrm{L}^{-1}$. Na voltametria de pulso diferencial o Nedocromil de Sódio apresentou dois picos catódicos, irreversíveis, nos valores de potencial de -0,67 V e $-0,75 \mathrm{~V}$ (vs ECS). Curvas analíticas foram obtidas no intervalo de $3,0 \times 10^{-6} \mathrm{~mol} \mathrm{~L}^{-1}$ a $1,0 \times 10^{-5} \mathrm{~mol} \mathrm{~L}^{-1}$, limite de detecção de $4,9 \times 10^{-7} \mathrm{~mol} \mathrm{~L}^{-1}$ e sensibilidade de $8,7 \times 10^{4} \mathrm{~mA} / \mathrm{mol} \mathrm{L}^{-1}$. Na voltametria de onda quadrada observou-se dois picos catódicos nos valores de potencial de $-0,80 \mathrm{~V}$ e $-0,91 \mathrm{~V}$ (vs ECS),

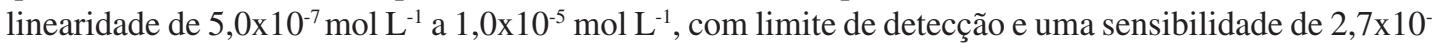
${ }^{6} \mathrm{~mol} \mathrm{~L}^{-1} \mathrm{e} 1,1 \times 10^{6} \mathrm{~mA} / \mathrm{mol} \mathrm{L}^{-1}$, respectivamente.

Palavras-chave: nedocromil de sódio; voltametria de varredura linear; voltametria de pulso diferencial; voltametria de onda quadrada; asma.

\section{Introdução}

Pertencente ao grupo das cromonas, o Nedocromil de Sódio vem sendo amplamente empregado para o tratamento da asma [7]. A ação das cromonas pode ser descrita como antiinflamatória e antialérgica . Este composto é usualmente administrado por inalação, sendo bem absorvido pelo pulmão, mas pouco absorvido pelo trato intestinal. O Nedocromil de Sódio não é metabolizado no corpo humano e a maior parte é excretada na urina. Ele inibe a geração de um derivado de neutrofila, e a consequente liberação de histamina na célula ${ }^{8}$, apresentando um excelente perfil de tolerabilidade, com efeitos colaterais pouco frequentes.<smiles>CCCc1c2oc(C(N)=O)cc(=O)c2cc2c(=O)cc(C(N)=O)n(CC)c12</smiles>

Figura 1. Fórmula Estrutural do Nedocromil de Sódio

O Nedocromil de Sódio contém dois grupos carbonilas aromáticos, redutíveis em potenciais de $-0,6 \mathrm{~V},-0,7 \mathrm{~V} \mathrm{e}-1,0 \mathrm{~V}$ em $\mathrm{pH} 3,0$, 
de acordo com a literatura [8]. Os estudos de farmacocinética e determinação deste composto têm sido realizados empregando-se técnicas convencionais de análise, como as técnicas cromatográficas [1,3]. Apesar da importância das técnicas eletroanalíticas na determinação de uma variedade de compostos de interesse farmacológico, há poucos trabalhos relacionados ao estudo eletroquímico do Nedocromil de Sódio, sendo estes realizados em eletrodo de mercúrio, $[6,8,10]$ com indesejáveis consequências toxicológicas. Assim sendo, o objetivo deste trabalho foi a determinação eletroanalítica do Nedocromil de Sódio, empregando as técnicas de voltametria de varredura linear, voltametria de pulso diferencial e voltametria de onda quadrada, em tampão B-R ( $\mathrm{pH} 4,0$ ), utilizando eletrodo de carbono vítreo.

\section{Procedimento experimental}

\section{Equipamentos}

As medidas voltamétricas foram realizadas em um Potenciostato/Galvanostato modelo 173 monitorado com um software M270, ambos da EG\&G PAR. Os estudos voltamétricos foram realizados em uma célula eletroquímica do tipo convencional com capacidade para $25 \mathrm{~mL}$, contendo um eletrodo de carbono vítreo com 0,07 $\mathrm{cm}^{2}$ de área, como eletrodo de trabalho (Tokai), um fio de platina como eletrodo auxiliar e um eletrodo de calomelano saturado (ECS) modelo GO115, da Analyon, como referência. O eletrodo de trabalho foi submetido a um tratamento prévio, o qual consiste de polimento com alumina (0,5 um), antes de cada medida voltamétrica.

\section{Soluções}

\section{Eletrólito Suporte}

A substância utilizada como eletrólito suporte foi o tampão Britton-Robinson ( $\mathrm{pH} 4,0) \mathrm{o}$ qual foi preparado pela adição das seguintes soluções: 0,04 mol L-1 de ácido ortofosfórico, 0,04 mol L ${ }^{-1}$ de ácido bórico, 0,04 $\mathrm{mol} \mathrm{L}^{-1}$ de ácido acético e $0,02 \mathrm{~mol} \mathrm{~L}^{-1}$ de hidróxido de sódio até que se atingisse o $\mathrm{pH}$ requerido.

Solução de Nedocromil de Sódio

Para os estudos voltamétricos do Nedocromil de
Sódio preparou-se uma solução padrão $1,0 \times 10^{-3}$ mol L-1 a partir do composto com grau de pureza analítico, tendo água deionizada como solvente.

\section{Procedimento}

\section{Voltametria de Varredura Linear}

Uma alíquota de $10 \mathrm{~mL}$ de solução tampão

$\mathrm{B}-\mathrm{R}$ foi adicionada à célula eletroquímica contendo um sistema de três eletrodos, sendo aplicado um fluxo de nitrogênio por um intervalo de quinze minutos para a remoção do oxigênio molecular eletroativo. Os voltamogramas para o estudo da dependência da corrente de pico catódica em relação à velocidade de varredura foram obtidos utilizando uma solução de concentração $1,0 \times 10^{-3}$ mol L ${ }^{-1}$ de Nedocromil de Sódio, sendo realizadas medidas voltamétricas no intervalo de 10 a 600 $\mathrm{mV} \mathrm{s}^{-1}$.

Posteriormente foi realizado um estudo da dependência da corrente de pico catódica em relação à concentração do analito. A dependência da corrente de pico catódica para o Nedocromil de Sódio foi investigada no intervalo de $5,0 \times 10^{-4}$ a $5,0 \times 10^{-3} \mathrm{~mol} \mathrm{~L}^{-1}$. Os diferentes valores de concentração para a espécie foram obtidos através de sucessivas adições de solução-estoque deste composto.

\section{Voltametria de Pulso Diferencial}

Um volume de $10 \mathrm{~mL}$ de solução do tampão B-R foi adicionada à célula eletroquímica, sendo esta solução desoxigenada por quinze minutos. Em seguida, os parâmetros operacionais, amplitude de potencial de pulso ( $\left.\mathrm{DE}_{\mathrm{p}}\right)$, tempo de duração do pulso $\left(\mathrm{t}_{\mathrm{p}}\right)$ e velocidade de varredura $(\mathrm{v})$, foram otimizados. Realizou-se o estudo da dependência da corrente de pico catódica em relação à concentração da espécie no intervalo de $3,0 \times 10^{-6}$ a $1,0 \times 10^{-5} \mathrm{~mol} \mathrm{~L}^{-1}$.

\section{Voltametria de Onda Quadrada}

Para os estudos do Nedocromil de Sódio por voltametria de onda quadrada foi utilizado um volume de $10 \mathrm{~mL}$ de solução tampão B-R, desoxigenada por 15 minutos contendo uma solução $1,0 \times 10^{-5} \mathrm{~mol} \mathrm{~L}^{-1}$ do fármaco. Foram inicialmente estudadas as condições ideais para os parâmetros operacionais de frequência (f) e 
magnitude de pulso aplicado $\left(\mathrm{E}_{\mathrm{SW}}\right)$. $\mathrm{O}$ estudo da dependência das correntes de pico catódicas em relação à concentração do Nedocromil de Sódio foi realizado no intervalo de $5,0 \times 10^{-7}$ a $1,0 \times 10^{-5}$ mol L-1.

\section{Resultados e discussão}

\section{Voltametria de Varredura Linear}

A voltametria constitui-se em uma técnica cuja origem remonta à polarografia, um tipo particular de voltametria, onde a diferença peculiar consiste no uso de um eletrodo gotejante de mercúrio (EGM) como eletrodo de trabalho. Dentre as técnicas voltamétricas, a modalidade de voltametria de varredura linear destaca-se pela simplicidade, onde uma variação de potencial é aplicada linearmente no intervalo de tempo estudado, sendo extensivamente utilizada no estudo de cinética eletródica e mecanismos reacionais, apresentando limites de detecção da ordem de $10^{-5}$ mol L-19.

O comportamento voltamétrico para a redução de solução $1,0 \times 10^{-3} \mathrm{~mol} \mathrm{~L}^{-1}$ de Nedocromil de Sódio, em tampão B-R ( $\mathrm{pH} 4,0$ ) apresentou dois picos catódicos, irreversíveis, com valores de potencial de pico correspondentes a $-0,86 \mathrm{~V}$ e $-1,10 \mathrm{~V}$ (vs ECS)

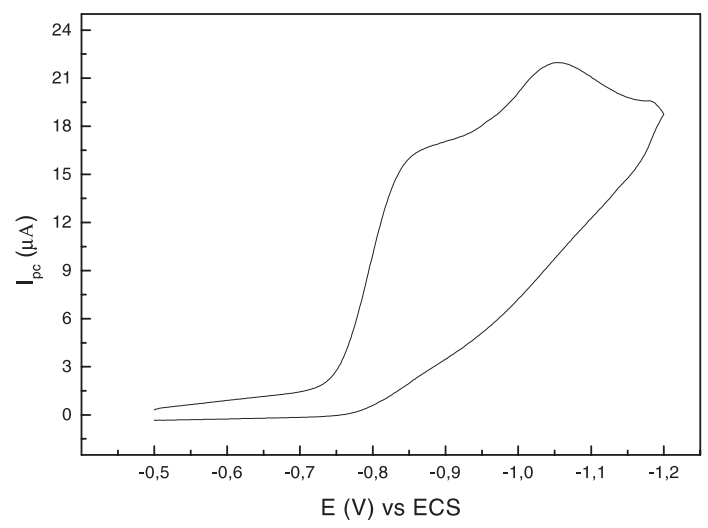

Figura 2. Voltamograma cíclico de solução $1,0 \times 10^{-3} \mathrm{~mol} \mathrm{~L}^{-1} \mathrm{de}$ Nedocromil de Sódio em tampão B-R (pH 4,0); v = 100,0 mV s .

A ausência de correntes de pico anódicas na varredura reversa de potencial e a variação dos valores de potencial de pico catódico $\left(\mathrm{E}_{\mathrm{pc}}\right)$, assim como $1 / 2 \mathrm{E}_{\mathrm{pc}}-\mathrm{E}_{\mathrm{pc} / 2} 1 / 2$, em função da velocidade de varredura, indicam que o processo eletródico apresenta um comportamento irreversível.

Tabela 1. Parâmetros ciclovoltamétricos da redução de solução 1,0x $10^{-3} \mathrm{~mol} \mathrm{~L}^{-1}$ de Nedocromil de Sódio, utilizando a técnica de Voltametria de Varredura Linear

\begin{tabular}{cccccc}
\hline & \multicolumn{2}{c}{ Primeiro Pico } & \multicolumn{2}{c}{ Segundo Pico } \\
\hline Velocidade & $\mathrm{E}_{\mathrm{pc} 1}$ & $\mathrm{E}_{\mathrm{p}}-\mathrm{E}_{\mathrm{p} / 2}$ & $\mathrm{E}_{\mathrm{pc} 2}$ & $\left|\mathrm{E}_{\mathrm{p}}-\mathrm{E}_{\mathrm{p} / 2}\right|$ \\
$\left(\mathrm{mV} \mathrm{s}^{-1}\right)$ & $(\mathrm{V})$ & $(\mathrm{V})$ & $(\mathrm{V})$ & $(\mathrm{V})$ \\
\hline 10 & $-0,838$ & 0,055 & $-1,047$ & 0,089 \\
20 & $-0,847$ & 0,067 & $-1,042$ & 0,078 \\
30 & $-0,864$ & 0,062 & $-1,068$ & 0,087 \\
40 & $-0,864$ & 0,063 & $-1,090$ & 0,066 \\
50 & $-0,859$ & 0,065 & $-1,057$ & 0,092 \\
100 & $-0,867$ & 0,068 & $-1,099$ & 0,064 \\
200 & $-0,893$ & 0,077 & $-1,111$ & 0,084 \\
400 & $-0,891$ & 0,073 & $-1,128$ & 0,080 \\
600 & $-0,906$ & 0,078 & $-1,121$ & 0,089 \\
\hline
\end{tabular}

Nos estudos da influência da velocidade de varredura na corrente de pico catódica $\left(\mathrm{I}_{\mathrm{pc}}\right)$ observou-se uma dependência linear de $\mathrm{I}_{\mathrm{pc}}$ com a raiz quadrada da velocidade, com coeficiente de correlação linear igual a 0,9993 evidenciando que o transporte de massa é controlado por difusão [4]. $\mathrm{Na}$ eletrorredução do Nedocromil de Sódio, os processos eletródicos envolvidos são devido à redução das funções alfa e beta insaturadas do grupo ceto a carbinols.

Considerando que o primeiro pico catódico apresenta melhores características para a

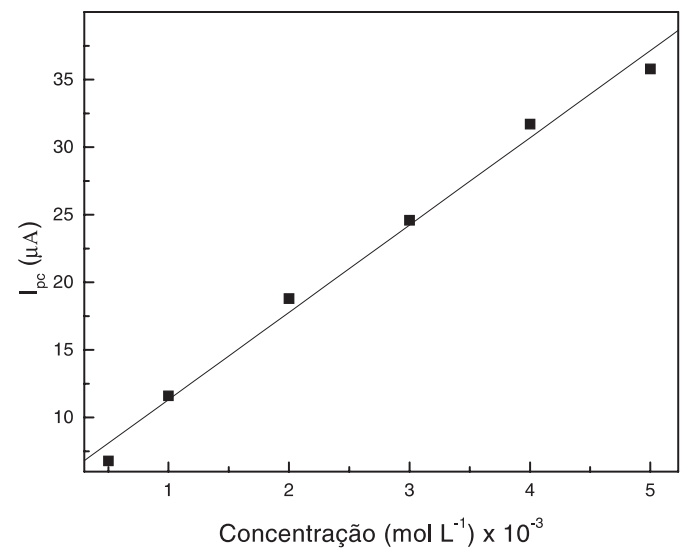

Figura 3. Influência da variação da concentração na corrente do pico catódico para solução de Nedocromil de Sódio, em tampão B-R ( $\mathrm{pH} 4,0)$, utilizando a técnica de voltametria de varredura linear $\left(\mathrm{v}=100,0 \mathrm{mV} \mathrm{s}^{-1}\right)$. 
determinação analítica deste fármaco, o gráfico de $I_{p c}$ versus a concentração para a solução de Nedocromil de Sódio, mostrou uma linearidade no intervalo de $5,0 \times 10^{-4}$ a $5,0 \times 10^{-3} \mathrm{~mol} \mathrm{~L}^{-1}$, com coeficiente de correlação linear de 0,9956 . O limite de detecção obtido foi de $8,2 \times 10^{-5} \mathrm{~mol} \mathrm{~L}^{-1}$, com uma sensibilidade amperométrica igual a $6,3 \times 10^{3}$ $\mathrm{mA} / \mathrm{mol} \mathrm{L}^{-1}$.

\section{Voltametria de Pulso Diferencial}

A voltametria de pulso diferencial é uma das técnicas de pulso mais importantes, onde a corrente é medida antes e depois da aplicação de um pulso de potencial. A altura correspondente à corrente de pico é proporcional à concentração da espécie oxidada/reduzida [2]. A voltametria de pulso diferencial fornece picos bem definidos e apresenta uma sensibilidade significativamente maior que os métodos voltamétricos clássicos com um limite de detecção na faixa de $10^{-7}$ a $10^{-8} \mathrm{~mol} \mathrm{~L}^{-19}$.

Foram realizados, inicialmente, estudos de otimização dos parâmetros operacionais da técnica de voltametria de pulso diferencial, como: amplitude de potencial de pulso aplicado $\left(\mathrm{DE}_{\mathrm{p}}\right)$, tempo de duração do pulso $\left(\mathrm{t}_{\mathrm{p}}\right)$ e velocidade de varredura (v). As condições ideais obtidas para estes parâmetros foram: $\mathrm{DE}_{\mathrm{p}}=100,0 \mathrm{mV}, \mathrm{t}_{\mathrm{p}}=10,0$ $\mathrm{ms}$ e $\mathrm{v}=5,0 \mathrm{mV} \mathrm{s}^{-1}$. Os voltamogramas obtidos para o Nedocromil de Sódio apresentaram dois picos catódicos, irreversíveis, nos valores de potencial $\left(\mathrm{E}_{\mathrm{pc}}\right)$ de $-0,67 \mathrm{~V},-0,75 \mathrm{~V}$ (vs ECS)

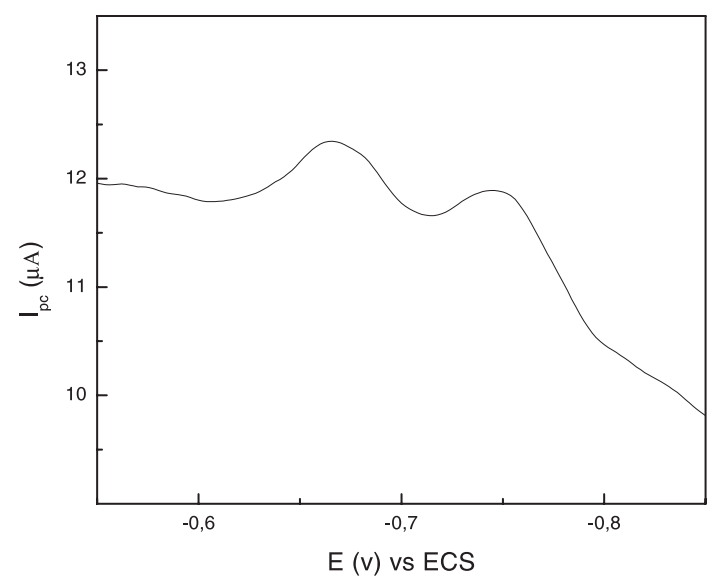

Figura 4. Voltamograma de pulso diferencial de solução $1,0 \times 10^{-5} \mathrm{~mol} \mathrm{~L}^{-1}$ de Nedocromil de Sódio em tampão B-R (pH $4,0) ; \Delta \mathrm{E}_{\mathrm{p}}=100,0 \mathrm{mV} ; \mathrm{t}_{\mathrm{p}}=10,0 \mathrm{~ms} ; \mathrm{v}=5,0 \mathrm{mV} \mathrm{s}^{-1}$.
Analisando o gráfico de $\mathrm{I}_{\mathrm{pc}}$ versus a concentração, da solução de Nedocromil de Sódio, observou-se uma linearidade no intervalo de 3,0x10${ }^{6} \mathrm{~mol} \mathrm{~L}^{-1}$ a $1,0 \times 10^{-5} \mathrm{~mol} \mathrm{~L}^{-1}$, com coeficiente de correlação linear de 0,9990 . O limite de detecção

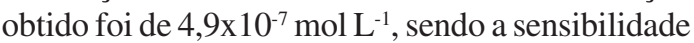
amperométrica equivalente a $8,7 \times 10^{4} \mathrm{~mA} / \mathrm{mol} \mathrm{L}^{-1}$, correspondente ao primeiro pico catódico o qual apresenta melhores características para a determinação analítica deste fármaco.

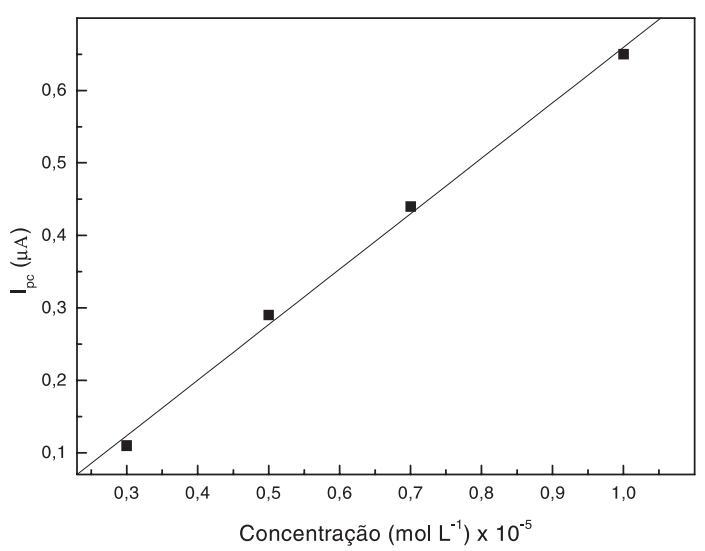

Figura 5. Influência da concentração na corrente de pico catódica para uma solução de Nedocromil de Sódio em tampão $\mathrm{B}-\mathrm{R}(\mathrm{pH} 4,0)$, empregando a técnica de voltametria de pulso diferencial. $\Delta \mathrm{E}_{\mathrm{p}}=100,0 \mathrm{mV} . \mathrm{t}_{\mathrm{p}}=10,0 \mathrm{~ms} . \mathrm{v}=5,0 \mathrm{mV} \mathrm{s}^{-1}$.

\section{Voltametria de Onda Quadrada}

$\mathrm{Na}$ voltametria de onda quadrada uma diferença de potencial de onda quadrada com uma pequena amplitude de pulso (1-50 mV) e uma frequiência de até $200 \mathrm{~Hz}$ é sobreposta a uma diferença de potencial que aumenta com o tempo [11]. A técnica é mais rápida e há um consumo menor de espécies eletroativas quando comparada com a voltametria de pulso diferencial. O limite de detecção é da ordem de $10^{-7}$ a $10^{-8} \mathrm{~mol} \mathrm{~L}^{-1}$. Como se faz a amostragem de corrente em ambos os pulsos, positivo e negativo, os picos correspondendo à oxidação ou à redução das espécies eletroativas na superfície do eletrodo podem ser obtidos na mesma experiência [5].

Inicialmente foram estudados os parâmetros operacionais de frequência (f) e magnitude de pulso aplicado $\left(\mathrm{E}_{\mathrm{SW}}\right)$. As melhores condições obtidas foram os valores de $150,0 \mathrm{~Hz}$ e $50,0 \mathrm{mV}$, respectivamente. 
A análise do voltamograma mostra dois picos catódicos nos valores de potencial $\left(\mathrm{E}_{\mathrm{pc}}\right) \mathrm{de}$ $0,80 \mathrm{~V}$ e $-0,91 \mathrm{~V}$.

A dependência de $\mathrm{I}_{\mathrm{pc}}$ com a concentração de Nedocromil de Sódio mostrou uma linearidade no intervalo de $5,0 \times 10^{-7}$ a $1,0 \times 10^{-5} \mathrm{~mol} \mathrm{~L}^{-1}$. Obtevese coeficiente de correlação linear igual a 0,9748 , um limite de detecção de $2,7 \times 10^{-6} \mathrm{~mol} \mathrm{~L}^{-1} \mathrm{e}$ sensibilidade amperométrica de $1,1 \times 10^{6} \mathrm{~mA} / \mathrm{mol}$ $\mathrm{L}^{-1}$, correspondente ao primeiro pico catódico o qual apresenta melhores características para a determinação analítica deste fármaco.
Na Tabela 2 estão reportados os parâmetros voltamétricos obtidos na determinação do Nedocromil de Sódio, utilizando eletrodo de carbono vítreo. Na determinação voltamétrica do fármaco observou-se que o maior intervalo de linearidade estudado foi obtido empregando a técnica de voltametria de onda quadrada a qual apresentou o maior valor de sensibilidade amperométrica. Entretanto, empregando a técnica de voltametria de pulso diferencial obteve-se o menor valor de limite de detecção.

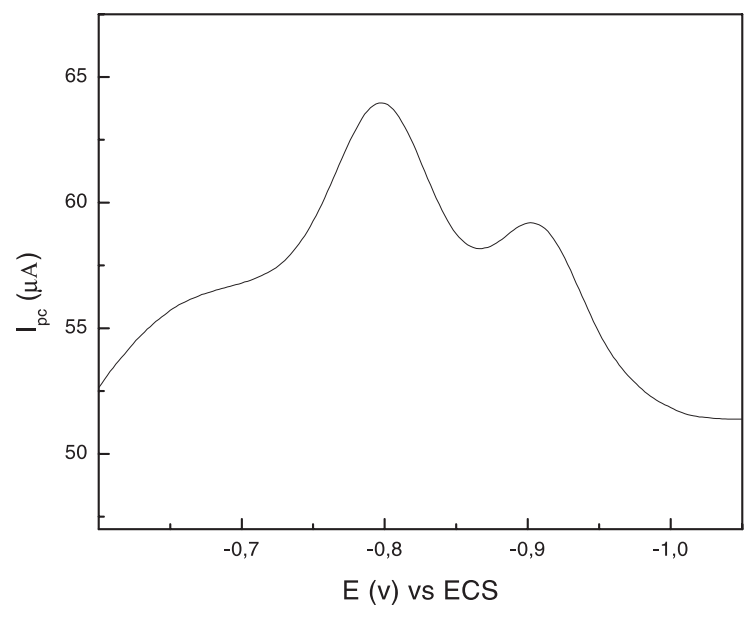

Figura 7. Influência da concentração na corrente do pico catódico para uma solução de Nedocromil de Sódio em tampão B-R (pH 4,0), empregando a técnica voltamétrica de onda quadrada. $\mathrm{f}=150,0 \mathrm{~Hz}$. $\mathrm{E}_{\mathrm{Sw}}=50,0 \mathrm{mV}$.

Tabela 2. Parâmetros voltamétricos para a determinação do Nedocromil de Sódio em eletrodo de carbono vítreo

\begin{tabular}{cccc}
\hline Parâmetros & VVL & VPD & VOQ \\
\hline Intervalo $\left(\mathrm{mol} \mathrm{L}^{-1}\right)$ & $5,0 \times 10^{-4}$ a $5,0 \times 10^{-3}$ & $3,0 \times 10^{-6}$ a $1,0 \times 10^{-5}$ & $5,0 \times 10^{-7}$ a $1,0 \times 10^{-5}$ \\
LD $(\mathrm{mol} \mathrm{L}-1)$ & $8,2 \times 10^{-5}$ & $4,9 \times 10^{-7}$ & $2,7 \times 10^{-6}$ \\
Sens. $\left(\mu \mathrm{A} / \mathrm{mol} \mathrm{L}^{-1}\right)$ & $6,3 \times 10^{3}$ & $8,7 \times 10^{4}$ & $1,1 \times 10^{6}$ \\
\hline
\end{tabular}

LD = Limite de Detecção; Sens. $=$ Sensibilidade amperométrica $; \mathrm{VVL}=$ Voltametria de Varredura Linear; VPD = Voltametria de Pulso Diferencial; VOQ = Voltametria de Onda Quadrada 


\section{Conclusões}

A voltametria de varredura linear, para a determinação eletroanalítica do Nedocromi de Sódio, em tampão B-R, apresentou uma boa sensibilidade amperométrica com pequeno limite de detecção, utilizando eletrodo de carbono vítreo. A técnica de pulso diferencial, quando comparada com a voltametria de onda quadrada, mostrou-se como a melhor técnica para a determinação voltamétrica do Nedocromil de Sódio por apresentar o menor limite de detecção e uma sensibilidade amperométrica adequada, em tampão B-R. Assim sendo, a voltametria de pulso diferencial poderia ser indicada para a determinação do Nedocromil de Sódio em estudos farmacológicos, onde a análise de concentrações menores é necessária.

\section{Agradecimentos}

Os autores agradecem o apoio financeiro da Fapesp.

T. F. Rosa, A. A. Saczk, M. V. B. Zanoni, N. R. Stradiotto. Voltammetric determination of necrodil sodium using glassy carbon electrode.

\begin{abstract}
Electroanalytical studies had been carried with the Nedocromil Sodium, an antiasthmatic drug, using the techniques of Linear Scan, Diferencial-Pulse and Square Wave Voltammetry. The technique of Linear Scan Voltammetry was used for determination of Nedocromil Sodium, at a glassy carbon electrode, in $\mathrm{pH} 4$ Britton-Robinson buffer. Two irreversible reduction peaks where obtained in the values of potential of $-0,86 \mathrm{~V}$ and $-1,1 \mathrm{~V}$. Calibration graphs were shown to be linear in the range $5,0 \times 10^{-4}-5,0 \times 10^{-3} \mathrm{~mol} \mathrm{~L}^{-1}$. The coefficients of linear correlation have been equal to 0,9990 , being the detection limit equal to $8,2 \times 10^{-5} \mathrm{~mol} \mathrm{~L}^{-1}$ and the amperometric sensitivity equal to $6,3 \times 10^{3} \mathrm{~mA} / \mathrm{mol} \mathrm{L}^{-1}$. Diferencial-Pulse Voltammetry two irreversible reduction peaks were obtained at the values of potential of $-0.67 \mathrm{~V}$ and $-0.75 \mathrm{~V}$. The study of the dependence of $\mathrm{I}_{\mathrm{pc}}$ with the concentration of the solution showed linearity in the range $3,0 \times 10^{-6}-1,0 \times 10^{-5}$

mol L-1, with coefficient of linear correlation of 0,9990 . The detection limit was calculated to be $4,9 \times 10^{-}$ ${ }^{7} \mathrm{~mol} \mathrm{~L}{ }^{-1}$, being the amperometric sensitivity equal to $8,7 \times 10^{4} \mu \mathrm{A} / \mathrm{mol} \mathrm{L}{ }^{-1}$. The technique of Square

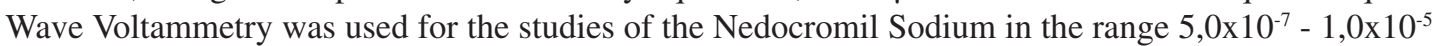
mol L ${ }^{-1}$. Two irreversible reduction peaks where obtained in the values of potential of $-0,80 \mathrm{~V}$ and $-0,91$ $\mathrm{V}$. The coefficients of linear correlation have been equal to 0,9748 , being the detection limit equal to
\end{abstract} $2,7 \times 10^{-6} \mathrm{~mol} \mathrm{~L}^{-1}$ and the amperometric sensitivity equal to $1,1 \times 10^{6} \mu \mathrm{A} / \mathrm{mol} \mathrm{L}^{-1}$.

Keywords: nedocromil sodium; linear scan voltammetry; diferencial-pulse voltammetry; square wave voltammetry; asthma.

\section{Referências bibliográficas:}

[1] O. A. Aswania, S. A. Corlett, H. B. Chrystyn. J. Chrom. 718 (2)(19980 290.

[2] P. W. Atkins. Físico-Química. vol. 3, 6 ed. Rio de Janeiro: Editora, 1999. p. 132-133.

[3] P. R. Baker, J. J. Gardner, D.Wilkinson. J. Chrom. 668 (1)(1995) 59.

[4] A. J. Bard, L. R. Faulhner. Electrochemical methods: fundamentals and applications. New York: Ed. John \& Sons, 1980. p. 718

[5] A. M. O. Brett, C. M. A. Brett, Eletroquímica princípios, métodos, e aplicações. Coimbra: Almedina, 1993. p.191-245. [6] H. Hoffmann, P. Hermening. Arch. Pharm. 325 (10)(1992) 643.

[7] T. S. C. Orr. J. Allergy Clin. Immunol. 88 (3)(1991) 313.

[8] R. Pirzard, J. C. Moreira, A. E. Davies, A. G. Fogg. Analyst 119 (11)(1994) 2439.

[9] D. A. Skoog, J. J. Leary. Principles of instrumental analysis. 4 ed., Saunders College Publishing, 1992. p. 555-56.

[10] M. V. B. Zanoni, J. C. Moreira, A. G. Fogg. Analyst 120 (2)(1995) 505.

[11] J. Zýka. Instumentation In Analytical Chemistry. vol. 1, Prague: Charles University, Ellis Horwood, 1991. p. 42-44. 